\title{
A Study on Iris Localization and Recognition on Mobile Phones
}

\author{
Kang Ryoung Park, ${ }^{1}$ Hyun-Ae Park, ${ }^{2}$ Byung Jun Kang, ${ }^{2}$ Eui Chul Lee, ${ }^{2}$ and Dae Sik Jeong ${ }^{2}$ \\ ${ }^{1}$ Department of Electronic Engineering, Biometrics Engineering Research Center, Dongguk University, 26 Pil-dong 3-ga, \\ Jung-gu, Seoul 100-715, South Korea \\ ${ }^{2}$ Department of Computer Science, Biometrics Engineering Research Center, Sangmyung University, Seoul 110-743, South Korea \\ Correspondence should be addressed to Kang Ryoung Park, viridity@freechal.com
}

Received 11 April 2007; Revised 3 July 2007; Accepted 30 August 2007

Recommended by N. V. Boulgouris

\begin{abstract}
A new iris recognition method for mobile phones based on corneal specular reflections (SRs) is discussed. We present the following three novelties over previous research. First, in case of user with glasses, many noncorneal SRs may happen on the surface of glasses and it is very difficult to detect genuine SR on the cornea. To overcome such problems, we propose a successive on/off dual illuminator scheme to detect genuine SRs on the corneas of users with glasses. Second, to detect SRs robustly, we estimated the size, shape, and brightness of the SRs based on eye, camera, and illuminator models. Third, the detected eye (iris) region was verified again using the AdaBoost eye detector. Experimental results with 400 face images captured from 100 persons with a mobile phone camera showed that the rate of correct iris detection was 99.5\% (for images without glasses) and 98.9\% (for images with glasses or contact lenses). The consequent accuracy of iris authentication was $0.05 \%$ of the EER (equal error rate) based on detected iris images.
\end{abstract}

Copyright (c) 2008 Kang Ryoung Park et al. This is an open access article distributed under the Creative Commons Attribution License, which permits unrestricted use, distribution, and reproduction in any medium, provided the original work is properly cited.

\section{INTRODUCTION}

Instead of traditional security features such as identification tokens, passwords, or personal identification numbers (PINs), biometric systems have been widely used in various kinds of applications. Among these biometric systems, iris recognition has been shown to be a highly accurate method of identifying people by using the unique patterns of the human iris [1].

Some recent additions to mobile phones have included traffic cards, mobile banking applications, and so forth. This means that it is becoming increasingly important to protect the security of personal information on mobile phones. In this sense, fingerprint recognition phones are already being manufactured. Other recent additions to these phones have been megapixel cameras. Our final goal is to develop an iris recognition system that uses only these built-in cameras and iris recognition software without requiring any additional hardware components such as DSP chips.

In addition to other factors such as image quality, illumination variation, angle of capture, and eyelid/eyelash obfuscation, the size of the iris region must be considered to ensure good authentication performance. This is because "the im- age scale should be such that irises with diameters will show at least 100 pixels diameter in the digital image to meet the recommended minimum quality level" [2]. In the past, it was necessary to use large zoom and focus lens cameras to capture images, so large iris images could not be obtained with small cheap mobile phones. However, a megapixel camera can make it possible to capture magnified iris images with no need for large zoom and focus cameras.

Even when facial images are captured relatively far away $(30 \sim 40 \mathrm{~cm})$, the captured regions possess sufficient pixel information for iris recognition. In addition, the cameraviewing angle is larger than in conventional iris cameras and the depth of field (DOF), in which focused iris images can be captured is larger, consequently. With captured facial images, eye regions must be detected for iris recognition. So, in this paper we propose a new iris detection method based on corneal specular reflections (SRs). However, for users with glasses, there may be many noncorneal SRs on the glasses and it can be very difficult to detect genuine SRs on the cornea. To overcome these problems, we also propose a successive on/off dual illuminator scheme.

Existing eye detection methods can be classified into two categories. Methods in the first category detect eyes based on 
the unique intensity distribution or the shape of the eyes under visual light [3-9]. Methods in the second category exploit the spectral properties of pupils under near IR illumination [10-12].

All the research discussed in [3-6] used a deformable template method to locate the human eye. The method discussed in [7] used multicues for detecting rough eye regions from facial images and performed a thresholding process. Rowley et al. [8] developed a neural network-based upright frontal facial feature (including the eye region) detection system. The face detection method proposed by Viola and Jones [9] used a set of simple features, known as an "integral image." Through the AdaBoost learning algorithm, these features were simply and efficiently classified and then a cascade of classifiers was constructed $[13,14]$.

In the method discussed in [10], eye detection was accomplished by simultaneously utilizing the bright/dark pupil effect under IR illumination and the eye appearance pattern under ambient illumination via the support vector machine (SVM). Ebisawa and Satoh. [11] generated bright/dark pupil images based on a differential lighting scheme that used two IR light sources (an on/off camera axis). However, it is difficult to use this method for mobile applications because the power of the light source must be very strong to produce a bright/dark pupil image (this increases the power consumption of mobile phones and reduces battery life). Also, large SRs can hide entire eye regions for users with glasses.

Suzaki [12] detected eye regions and checked the quality of eye images by using specular reflections for racehorse and human identification. However, the magnified eye images were captured close to the object in an illuminatorcontrolled harness place. This led to small noncorneal SR regions in the input image. Also, these researchers did not consider users with glasses. In addition, they only used heuristic experiments to determine and threshold the size and pixel intensity value of the SR in the image. In [15], the activation/deactivation illuminator scheme was proposed to detect eye regions based on corneal SRs. However, because these researchers used a single illuminator, detection accuracy was degraded when there were many noncorneal SRs on the surface of glasses. In addition, because eye regions were determined only based on detected SRs, there were many false acceptance cases, which meant that noneye regions were falsely regarded as eye regions. Also, only the iris detection accuracy and processing times were shown. In [16], the researchers also used the on/off illuminator scheme, but it was used for detecting rough eye positions for face recognition.

In [17], the researchers proposed a method for selecting good quality iris images from a sequence based on the position and quality of the SR relative to the pupil. However, they did not solve the problem of detecting corneal SRs when there were many noncorneal SRs when users wore glasses. In addition, they did not show the theoretical size and brightness of corneal SRs.

To overcome these problems, we propose a rapid iris detection method for use in mobile phones and based on SRs. To determine the size and pixel intensity values of the SRs in the image, theoretically, we considered the eye model and the camera, the eye, and the illuminator geometry. In addition, we used a successive on/off dual illuminator to detect genuine SRs (in the pupil region) for users with glasses. Also, we excluded the floating-point operation to reduce processing time, since the ARM CPU used in mobile phones does not have floating-point coprocessors.

\section{PROPOSED IRIS DETECTION ALGORITHM}

\subsection{Overview of the proposed method and the illuminator on/off scheme}

An overview of the proposed method is shown in Figure 1 [16]. First, the user initiates the iris recognition process by clicking the "start" button of a mobile phone. Then, the camera microcontroller alternatively turns on and off the dual (left and right) infra-red (IR) illuminators. When only the right IR illuminator is turned on, two facial images (Frame $\# 1$, \#2) are captured, as shown in Figure 2. And then, another one (Frame \#3) is captured when both illuminators are turned off. After that, two additional facial images (Frame $\# 4, \# 5$ ) are captured again when only the left IR illuminator is turned on. So, we obtained five successive images as shown in Figures 1(1) and 2. This scheme was iterated successively as shown in Figure 2. When Frames \#1-\#5 did not meet our predetermined threshold for motion and optical blurring (as shown in Figure 1(2), (3)), another five images (Frame \#6\#10) were used (Figure 1(4)).

The size of the original captured image was $2048 * 1536$ pixels. To reduce processing time, image was $2048 * 1536$ pixels. To reduce processing time, we used the eye region in a predetermined area of the input image. Because we attached a cold mirror (to pass the IR light through and reflect the visible light) in front of the camera lens and the eye-aligning region was indicated on the mirror as shown in Figure 5, the user was able to align his or her eye with the camera. So, the eye existed in the restricted region of any given captured image. This kind of eye-aligning scheme has been adopted by conventional iris recognition cameras such as the LG IrisAccess 3000 or the Panasonic BM-ET300. By using the eyealigning region in the cold mirror, we were able to determine that eye regions existed in the area of $(0,566) \sim(2048,1046)$ in the input image. So, it was not necessary to process the whole input image $(2048 \times 1536$ pixels $)$ and we are able to reduce processing time. For this, the captured eye region images $(2048 \times 480$ pixels $((0,566) \sim(2048,1046)))$ were $1 / 6$ down-sampled $(341 \times 80$ pixels image $)$ and we checked the amount of motion blurring in the input image as shown in Figure 1(2).

In general, the motion blur amount (MBA) can be calculated by the difference image between two illuminator-on images. If the calculated MBA was greater than the predetermined threshold ( $\boldsymbol{T h} \mathbf{l}$ as shown in Figure 1) (we used 4 as a threshold), we determined that the input image was too blurred to be recognized. After that, our system checked the optical blurring amount (OBA) by checking the focus values of the A2 and A4 images in Figure 2, as shown in Figure 1(3). In general, focused images contain more highfrequency components than defocused images [18]. We used the focus checking method proposed by Kang and Park [19]. 


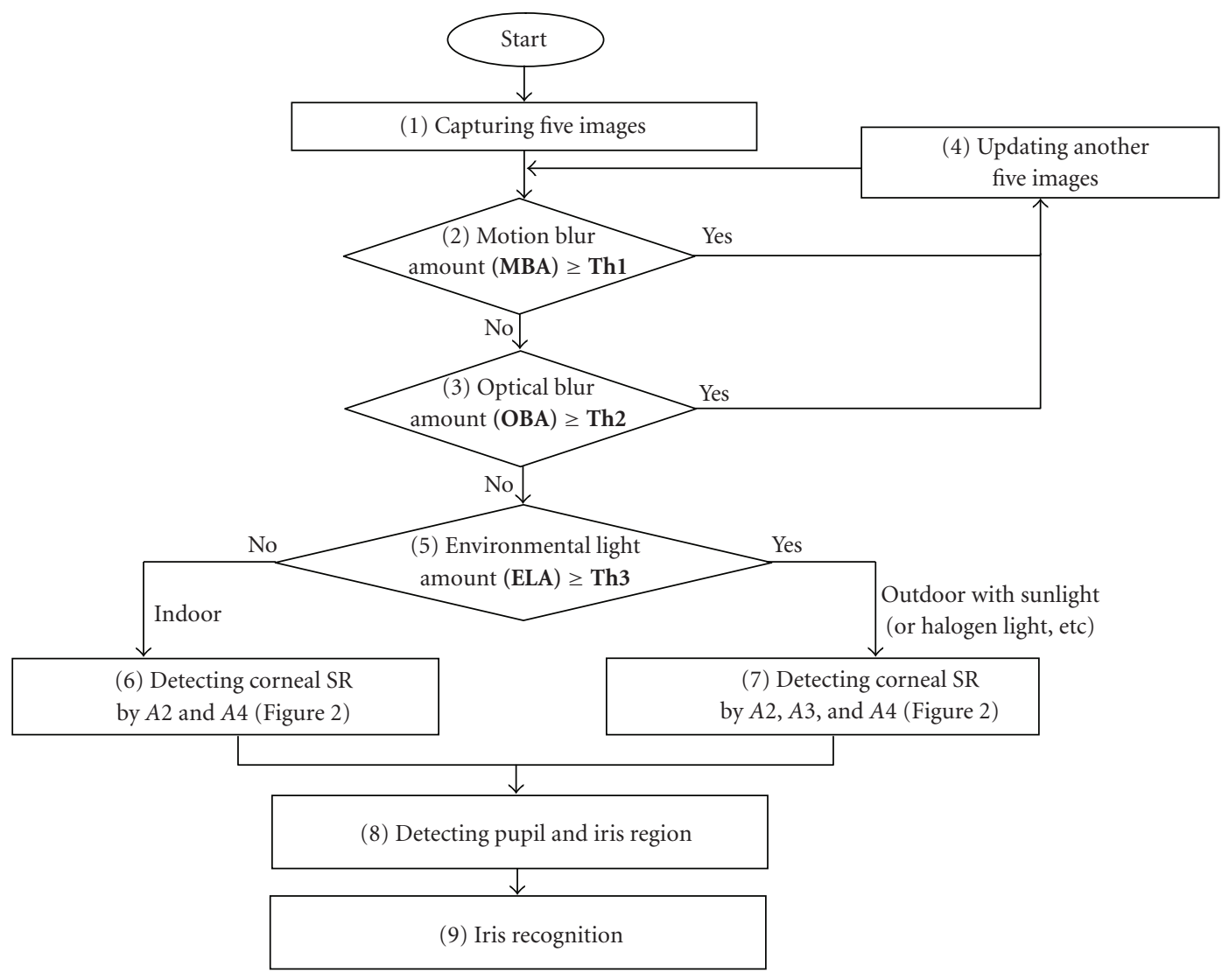

FIGURE 1: Flowchart of the proposed method.

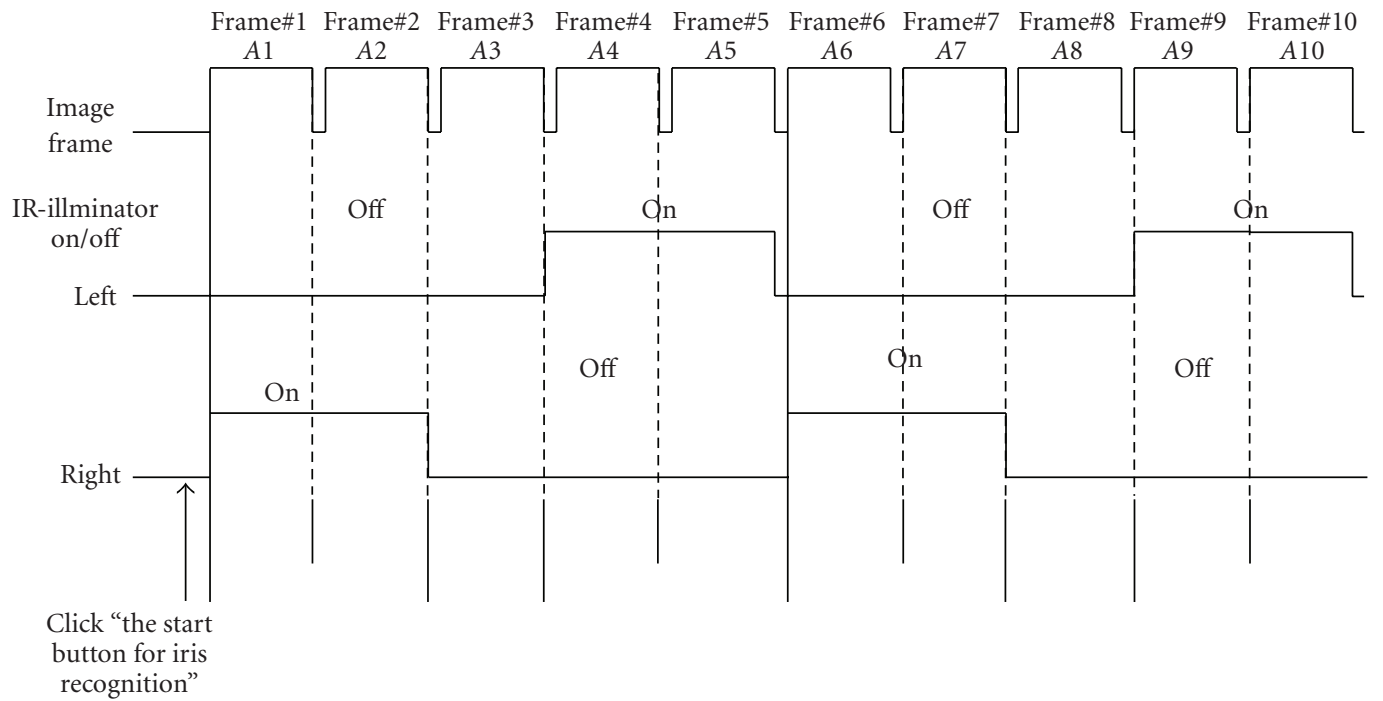

FIGURE 2: The alternative on/off scheme of the dual IR-illuminators [16].

The calculated focus value was compared to the predetermined threshold. If all the focus values of $\mathrm{A} 2$ and $\mathrm{A} 4$ were not below the threshold (Th2 as shown in Figure 1) (we used 70 as the threshold), we regarded the input image as defocused and captured five other images as shown in Figure 1(4), as mentioned before.
Next, our system calculated the environmental light amount (ELA) of the illuminator-off image (the average gray level of A3 shown in Figure 2) to check whether outer sunlight existed or not in the input image, as shown in Figure 1(5). As shown in Figure 5, we attached a cold mirror with an IR-Pass filter in front of the camera lens so that 
image brightness was not affected by visible light. In indoor environments, the average gray level of the illuminator-off image (A3) was very low (our experiments showed that it was below 50 (Th3)).

However, sunlight includes a large amount of IR light and in outdoor environments, the average gray level of the illuminator-off image (A3) increases (more than 50 (Th3)).

The American Conference of Government Industrial Hygienists (ACGIH) exposure limit for infrared radiation is defined by the following equation. For exposures greater than 1,000 seconds, irradiance must be limited to less than $10 \mathrm{~mW} / \mathrm{cm}^{2}$ [20],

$$
\sum_{770 \mathrm{~nm}}^{3000 \mathrm{~nm}} E_{\lambda} \bullet \Delta \lambda \leq 1.8 t^{-3 / 4} \mathrm{~W} / \mathrm{cm}^{2},
$$

where $\lambda$ represents the wavelength of incident light, $E_{\lambda}$ represents the irradiance onto the eye in watts $/ \mathrm{cm}^{2}$, and $t$ represents the exposure time in seconds. In our iris recognition system, the exposure time $(t)$ was a maximum of five seconds (time-out) for enrollment or recognition. We obtained the maximum ACGIH exposure limits for infrared radiation as $540 \mathrm{~mW} / \mathrm{cm}^{2}$ based on (1). As shown in Section 2.2, the $Z$-distance between the illuminator and the eye in our system was $250-400 \mathrm{~mm}$. Experimental results showed that the infrared radiation power $\left(0.44 \mathrm{~mW} / \mathrm{cm}^{2}\right)$ of our system was much less than the limits $\left(540 \mathrm{~mW} / \mathrm{cm}^{2}\right)$, so it met the safety requirements.

\subsection{Detecting corneal SRs by using the difference image}

After that, our system detected the corneal specular reflections in the input image. For indoor environments (ELA < Th3 shown in Figure 1(6)), corneal SR detection was performed using the difference image between A2 and A4 in Figures 1(6) and 3. In general, large numbers of noncorneal SRs (with similar gray levels to genuine SRs on the cornea) occurred for users with glasses and that made it difficult to detect genuine SRs on the cornea (inside the pupil region, as shown in Figure 3). So, we used a difference image to detect the corneal SRs easily. That is because the genuine corneal SRs had horizontal pair characteristics in the difference image as shown in Figure 3(c) and their interdistance in the image was much smaller than that of other noncorneal SRs on the surface of glasses. Also, the curvature radius of the cornea was much smaller than that of glasses. However, in outdoor environments, SR detection was performed using the difference image between $((\mathrm{A} 2-\mathrm{A} 3) / 2+127)$ and $((\mathrm{A} 4-\mathrm{A} 3) / 2+127)$, as shown in Figure 1(7).

In outdoor environments, the reason we used $\mathrm{A} 2-\mathrm{A} 3$ and $A 4-A 3$ was to get rid of the effect of sunlight. A3 was only illuminated by sunlight. So, by obtaining the difference image between A2 and A3 (or A4 and A3), we were able to reduce the effect of sunlight. In detail, in outdoor environments, sunlight increased the ELA. So, in addition to the corneal SR, the brightness of other regions such as the sclera and facial skin became so high (their brightness became similar to that of the corneal SR) that it was very difficult to dis-

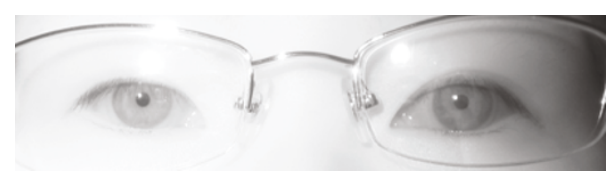

(a)

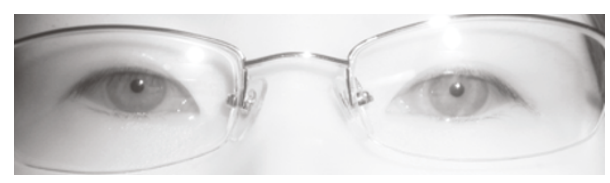

(b)

Imposter SRs on the glasses surface and frame

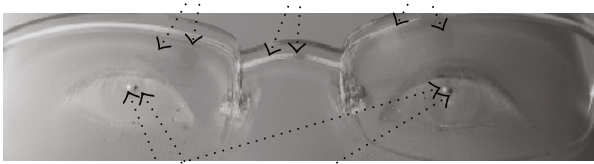

Genuine corneal SR (gray level is over 250)

Genuine corneal SR (gray level is below 4)

(c)

FIGURE 3: The captured eye images for users with glasses. (a) Eye image with right illuminator on, (b) eye image with left illuminator on, and (c) difference image between (a) and (b).

criminate those regions from the corneal SR only by using the difference images of A2 and A4 like (6) of Figure 1.

In this case, because the effect of sunlight was included in both A2 and A4, by subtracting the brightness of A3 (because it was captured with the camera illuminator off, its brightness was determined only by outer sunlight) from A2 and A4 $(((\mathrm{A} 2-\mathrm{A} 3) / 2+127)$ and $((\mathrm{A} 4-\mathrm{A} 3) / 2+127))$, we got rid of the effect of sunlight in A2 and A4. Consequently, the brightness of other regions such as sclera or facial skin regions became much lower compared to that of the corneal SR and we were easily able to discriminate the corneal SR from other regions.

Based on that, we used the following three pieces of information to detect the genuine SRs inside the pupil region. First, the corneal SR is small and it can be estimated by the camera, eye, and illuminator models (details are shown in Section 3). Second, genuine corneal SRs have horizontal pair characteristics in the difference image that are different from other noncorneal SRs on the surface of glasses because they are made by left and right illuminators. Since we knew the curvature radius of the cornea $(7.8 \mathrm{~mm})$ based on Gullstrand's eye model [21], the distance $(50 \mathrm{~mm})$ between the left and right illuminators and the $Z$-distance was 250$400 \mathrm{~mm}$. Also, our iris camera had an operating range of 250-400 $\mathrm{mm}$ between the eye and the camera, and we were able to estimate the pixel distance (on the $X$ axis) between the left and right genuine SRs in the image based on the perspective projection model [22].

Especially, because the curvature radius of the cornea is much smaller than that of the surface of the glasses, the distance between the corneal left and right SRs is shorter than that between the noncorneal ones in the image, as shown in Figure 3. However, because there was a time difference be- 
tween the left and right SR images (as shown in Figure 2, the time difference of A2 and A4 is 66 milliseconds) and there was also hand vibration, there was also a vertical disparity of the left and right SR positions. Experimental results showed a maximum of \pm 22 pixels in the image (which corresponds to the movement of $0.906 \mathrm{~mm}$ per 66 milliseconds as measured by the Polhemus FASTRAK [23]) and we used it as the vertical margin of the left and right SR positions.

Third, because genuine SRs occur in the dark pupil region (whose gray level is below 5) and its gray level is higher than 251 (see Section 4), the difference value $(=(\mathrm{A} 2-\mathrm{A} 3) / 2+127$ in indoor environments $)$ of the genuine SR is higher than 250 or lower than 4. Also, using a similar method, we estimated the difference value $(=((\mathrm{A} 2-\mathrm{A} 3) / 2+127)-((\mathrm{A} 4-\mathrm{A} 3) / 2+127))$ of the genuine corneal SRs in outdoor environments. Based on that, we discriminated the genuine SR from the noncorneal ones. From the difference image, we obtained the accurate center position of the genuine SRs based on the edge image obtained by the $3 \times 3$ Prewitt operator, component labeling, and circular edge detection. Based on the detected position of the genuine SR in the 1/6 down-sampled image, pupil, iris detection, and iris recognition were performed in the original image (details are shown in Sections 5 and 6).

\section{ESTIMATING THE SIZE OF CORNEAL SPECULAR REFLECTIONS IN IMAGES}

\subsection{Size estimation of SRs in focused images}

In this section, we estimate the size of the genuine SRs on the cornea based on eye, camera, and illuminator models as shown in Figure 4 [15]. Previous researchers [12] have used only heuristic experiments to determine and threshold the size and pixel intensity values of the SRs in images. Also, in this section, we discuss why the SRs are brighter than the reflection of the skin.

By using the Fresnel formula $(\rho=(n 1-n 2) /(n 1+n 2)$, where $\rho$ is the reflection coefficient, $n 1$ is the refractive index of the air $(=1)$, and $n 2$ is that of the cornea (=1.376) [21] (or facial skin (=1.3) [24])), we obtained the reflection coefficients $(\rho)$ of the cornea as about -0.158 (here, the reflectance rate is $2.5\left(=100^{*} \rho^{2}\right)$ ) and the skin as about -0.13 (here, the reflectance rate is 1.69). So, we discovered that the SRs are brighter than the reflection of the skin.

We then tried to estimate the size of the SR in the image. In general, the cornea is shaped like a convex mirror and it can be modeled as shown in Figure 4 [25]. In Figure 4, $\mathrm{C}$ is the center of the eyeball. The line that passes from the cornea's surface through $\mathrm{C}$ is the principal axis. The cornea has a focal point F, located on the principal axis. According to Gullstrand's eye model [21] and the fact that $\mathrm{C}$ and $\mathrm{F}$ are located on the opposite sides of the object, the radius of the cornea $(R)$ is $-7.8 \mathrm{~mm}$ and the corneal focal length $\left(f_{1}\right)$ is $-3.9 \mathrm{~mm}$ (because $2 * f_{1}=R$ in the convex mirror). Based on that information, we obtained the image position $(b)$ of the reflected illuminator by $\left(1 / f_{1}=1 / a+1 / b\right)$. Here, $a$ represents the distance between the cornea surface and the camera illuminator. Because our iris camera in the mobile phone had an operating range of $25-40 \mathrm{~cm}$, we defined $a$ as $250-$ $400 \mathrm{~mm}$. From that, $b$ was calculated as $-3.84-3.86 \mathrm{~mm}$ and we used $-3.85 \mathrm{~mm}$ as the average value of $b$. From that calculation, we obtained the image size of the reflected illuminator $\left(\overline{A^{\prime} B^{\prime}}\right)\left(\because \overline{A^{\prime} B^{\prime}} / \overline{A B}=b / a\right.$ as shown in Figure 4$)$ as $0.096-0.154 \mathrm{~mm}$, because $\overline{A B}$ (the diameter of the camera illuminator) was $10 \mathrm{~mm}$. We then adopted the perspective model between the eye and the camera and obtained the image size $(X)$ of the SR in the camera, as shown in Figure 4(a) $\left(a+|b|: \overline{A^{\prime} B^{\prime}}=f_{2} c: \mathrm{X}, \mathrm{X}\right.$ is $1.4-3.7$ pixels in the image). Here, $f_{2}$ (the camera focal length) was $17.4 \mathrm{~mm}$ and $c$ (the distance between the CCD cell) was $349 \mathrm{pixel} / \mathrm{mm} . f_{2}$ and $c$ were obtained by camera calibration [22]. Consequently, we determined the size (diameter) of the SR as 1.4-3.7 pixels in the focused input image and used that value as a threshold for size filtering when detecting the genuine SR on the cornea.

However, in one case, the user tried to identify his iris by holding the mobile phone, which led to image blurring. This blurring by hand vibration occurs frequently and it increases the image size of the SR (by optical and motion blurring). When this happens, we also need to consider the blurring to determine the image size of the SR.

The meaning to estimate the size of corneal SR is like this. Based on Figure 4, we were able to estimate the size of the corneal SR theoretically by not capturing actual eye images including the corneal SR. Of course, by using heuristic methods, we were able to estimate the size of the corneal SR. But for that, we had to obtain many images and analyze the size of the corneal SR intensively. In addition, most conventional iris cameras include the $Z$-distance measuring sensor with which $a$ of Figure 4 can be obtained automatically. In this way, the size of the corneal SR can be estimated easily without requiring intensive and heuristic analysis of many captured images. The obtained size information can be used for size filtering in order to detect the corneal SR among many noncorneal SRs.

In order to prove our theoretical model, we used 400 face images captured from 100 persons (see Section 7). Among them, we extracted the images which were identified by our iris recognition algorithm (because the size (1.4-3.7 pixels) of the SR denoted a focused image). Then, we measured the size of the SR manually and found that the obtained size of the SR was almost the same as that obtained theoretically.

Because the corneal SR was generated on the cornea mirror surface as shown in Figure 4 and it was not reflected on the surface of the glasses, the actual size of the SR did not change irrespective of wearing glasses. Of course, many noncorneal SRs occurred on the surface of the glasses. To prove this, we analyzed the actual SR size with the images of glasses among 400 face images and we found that the size of SR was not changed when glasses were worn.

\subsection{Optical blur modeling of SRs}

In general, optical blurring can be modeled as $(O(u, v)=$ $H(u, v) \bullet I(u, v)+N(u, v)$, where $O(u, v)$ represents the Fourier transform of the blurred iris image caused by defocusing, $H(u, v)$ represents that of the degradation function (2-D PSF), $I(u, v)$ represents that ofthe clear (focused) image, and $N(u, v)$ represents that of noise [22]). In general, 


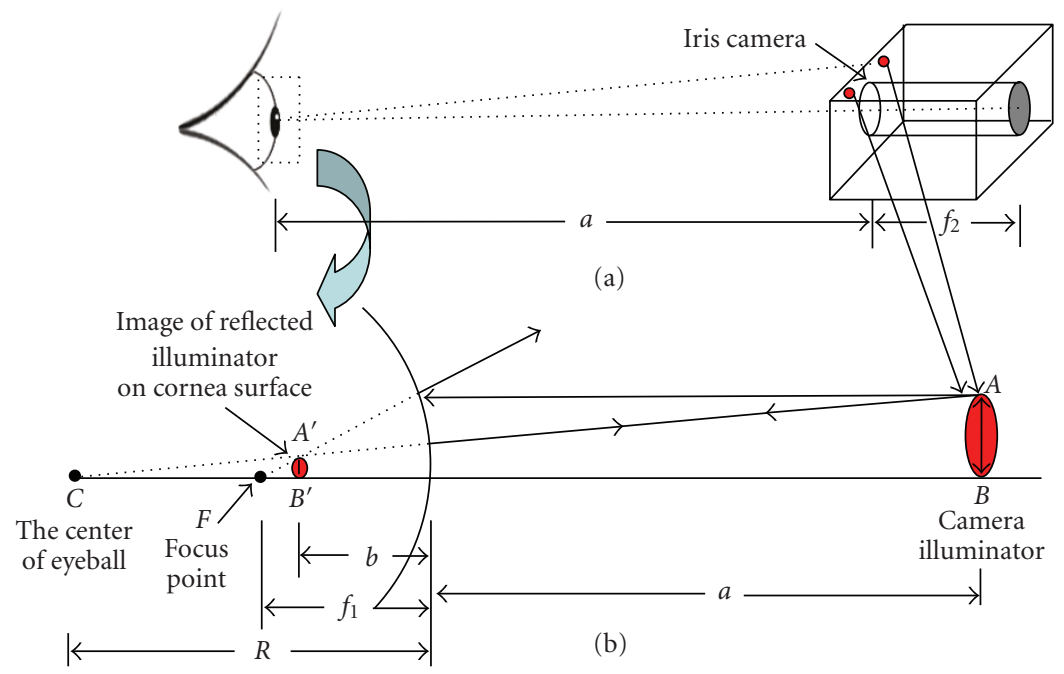

FIgURE 4: A corneal SR and the camera, illuminator, eye model [15]. (a) The camera, illuminator, and eye model, (b) a corneal SR in the convex mirror.

$N(u, v)$ is much smaller than other terms and can be excluded. Because the point spread function (PSF) $(H(u, v))$ of optical blurring can be represented by the Gaussian function [22], we used the Gaussian function for it.

To determine an accurate Gaussian model, we obtained the SR images at a distance of $25 \sim 40 \mathrm{~cm}$ (our operating range) in the experiment. We then selected the best focused SR image as $I(u, v)$ and the least focused one as $O(u, v)$. With those images, we determined the mask size and variance of the Gaussian function $(H(u, v))$ based on inverse filtering [22]. From that, we determined that the maximum size (diameter) of the SR was increased to $4.4 \sim 6.7$ pixels in the blurred input image (1.4-3.7 pixels in the focused image). We used those values as a threshold for size filtering when detecting the genuine SR [15].

\subsection{Motion blur modeling of SRs}

In addition, we considered motion blurring of the SRs. In general, motion blurring is related to the shutter time of the camera lens. The longer the shutter time, the brighter the input image, but the more severe the degree of motion blurring. In these cases, the SRs are represented by ellipses instead of circles. To reduce motion blurring, we could have reduced the shutter time, but the input image was too dark to be used for iris recognition. We could also have used a brighter illuminator, but this may have led to an increase of system costs. Due to these reasons, we set our shutter time as $1 / 30$ second (33 milliseconds).

To measure the amount of motion blurring by a conventional user, we used a 3D position tracker sensor (Polhemus FASTRAK [23]). Experimental results showed that translations in the directions of the $X, Y$, and $Z$ axes were $0.453 \mathrm{~mm}$ per 33 milliseconds. From that information, and based on the perspective model between the eye and the camera as shown in Figure 4(a), we estimated the ratio between the vertical and horizontal diameters of the SR, the maximum length of the major SR axis, that of the minor axis, and the maximum SR diameter in the input image. We used those values as the threshold for shape filtering when detecting the genuine SR. Even if we used another kind of iris camera, we knew $a, f_{2} c$, and $\overline{A B}$ as shown in Section 3.1 (as obtained by camera calibration or the camera and illuminator specifications). So, we obtained the above size and shape information of the SR irrespective of the kind of iris camera [15].

\section{ESTIMATING THE INTENSITY OF CORNEAL SPECULAR REFLECTIONS IN IMAGES}

The Phong model identifies two kinds of light (ambient light and point light) [26]. However, because we used a cold mirror (IR pass filter) in front of the camera as shown in Figure 5, we were able to exclude the effect of ambient light when estimating the brightness of the SR. Although point light has been reported to produce both diffuse elements and SRs, only SRs can be considered in our modeling of corneal SRs, as shown in (2),

$$
L=\frac{I_{p}\left[K_{s}(V \cdot R)^{n}\right]}{d+d_{0}},
$$

where $L$ is the reflected brightness of the SR, $R$ is the reflected direction of incident light, and $V$ is the camera viewing direction. $K_{s}$ is the SR coefficient, as determined by the incident angle and the characteristics of the surface material. Here, the distance between the camera and the illuminator was much smaller than the distance between the camera and the eye as shown in Figure 4(a). Due to that, we supposed that the incident angle was about 0 degrees. Also, the angle between $V$ and $R$ was 0 degree (so, $V \cdot R=1$ ). From that, $K_{s}$ was only represented as the reflection coefficient $(\rho)$ of the cornea as about -0.158 . This value was obtained in Section 3.1. $I_{p}$ represents the power of incident light (camera illuminator) measured as 620 lux. $d$ is the operating range $(250-400 \mathrm{~mm})$ and $d_{0}$ is the offset term (we used $5 \mathrm{~mm}$ ) to ensure that the di- 


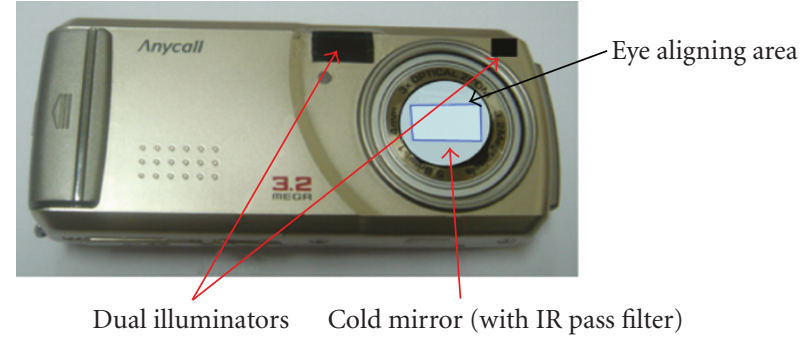

FIGURE 5: Mobile phone used for iris recognition.

vider did not become $0 . n$ represents the constant value, as determined by the characteristics of the surface. From that, we obtained $L$ (the SR reflected brightness on the cornea surface) as $0.242-0.384 \mathrm{lux} / \mathrm{mm}$. From (2) and (3), we obtained the radiance $L^{\prime}\left(0.0006-0.0015 \mathrm{lux} / \mathrm{mm}^{2}\right)$ of the SR into the camera:

$$
L^{\prime}=\frac{L}{d+d_{0}^{\prime}},
$$

where $d$ is the distance between the camera and the eye, and $d_{0}^{\prime}$ is the offset term of $5 \mathrm{~mm}$. We then obtained the image irradiance $E$ value of the SR [27]:

$$
E=L^{\prime}\left(\frac{\pi}{4}\right)\left(\frac{D}{f}\right)^{2} \cos ^{4} \alpha
$$

where $f$ and $D$ represent the camera focal length $(17.4 \mathrm{~mm})$ and aperture of the lens $(3.63 \mathrm{~mm})$, respectively, [28]. $\alpha$ is the angle between the optical axis and the ray from the center of the SR to the center of the lens. Because the distance between the optical axis and the SR is much smaller than the distance between the camera and the eye as shown in Figure 4(a), we supposed $\alpha$ was 0 degree. From that, we found that $E$ was $2.05 \times 10^{-5} \sim 5.12 \times 10^{-5} \mathrm{lux} / \mathrm{mm}^{2}$. Finally, we obtained the image brightness of the corneal SR $(B)[27]$ :

$$
B=F(E t c)=(E t c)^{\gamma},
$$

where $t$ is the camera shutter time, and $c$ is the auto gain control (AGC) factor. In general, $\gamma$ can be assumed to be 1. In our camera specifications, $t$ is 33 milliseconds and $c$ is $3.71 \times 10^{5} \mathrm{~mm}^{2} /$ Lux $\cdot$ milliseconds. From those values, we obtained the minimum intensity of the corneal SR in the image as 251 and used it as the threshold value to detect the corneal SR. Even if we used another kind of iris camera, we obtained the above camera and illuminator parameters by camera calibration or camera and illuminator specifications. Therefore, we obtained the minimum intensity of the corneal SR in the image irrespective of the kinds of iris camera hardware [15].

\section{PUPIL AND IRIS DETECTION AND VERIFICATION WITH THE ADABOOST CLASSIFIER}

Based on the size, shape, and brightness of the SR obtained from theoretical analysis in Sections 3 and 4, we were able to detect the accurate SR position of the pupil in the difference image by the method mentioned in Section 2.2. After that, before detecting the pupil region based on the detected SR, we verified the detected eye region by using the AdaBoost algorithm [9]. That is because when there are large SRs on the surface of glasses caused by left or right illuminators, it is possible not to detect accurate SR positions in the pupil.

The original AdaBoost classifier used a boosted cascade of simple classifiers with Haar-like features capable of detecting faces in real time at both high detection rates and very low false positive rates $[13,14]$. In essence, the AdaBoost classifier represents a sequential learning method based on a one-step greedy strategy. It is reasonably expected that postglobal optimization processing will further improve AdaBoost performance [13]. A cascade of classifiers is a decision tree where at each stage a classifier is trained and formed to detect almost all objects while rejecting a certain percentage of background areas. Those image windows not rejected by a stage classifier in the cascade sequence will be processed by the successful stage classifiers [13]. The cascade architecture can dramatically increase the speed of the detector by focusing attention on promising regions. Each stage classifier was trained by the AdaBoost algorithm $[13,29]$. The idea of boosting refers to selecting a set of weak learners to form a strong classifier [13].

We modified the original AdaBoost classifier for verification of detected eye regions by using corneal SRs. For training, we used 200 face images captured from 70 persons and in each image, we selected the eye and noneye regions manually for classifier training. Because we applied the AdaBoost classifier only to the detected eye candidate region by using the SRs, it did not take much processing time (less than 0.5 milliseconds when using a Pentium-IV PC (3.2 Ghz)). Then, if the detected eye region was correctly verified by the AdaBoost classifier, we defined the pupil candidate box as $160 * 160$ pixels based on the detected SR position. Here, the box size was determined by the human eye model. The conventional size of the pupil was adjusted from $2 \mathrm{~mm}$ to $8 \mathrm{~mm}$ depending on the level of extraneous environmental light [30]. The magnification factor of our camera was 19.3 pixels $/ \mathrm{mm}$. Consequently, we estimated the pupil diameter from 39 to 154 pixels in the input image ( $2048 * 480$ pixels). The size of the pupil candidate box was determined to be $160 * 160$ pixels (in order to cover the pupil at the maximum size).

Then, in the pupil candidate box, we applied circular edge detection to detect accurate pupil and iris regions $[1,31]$. To enhance processing speed, we used an integerbased circular edge detection method, which excluded the floating-point operation [32].

\section{IRIS RECOGNITION}

To isolate iris regions from eye images, we performed pupil and iris detection based on the circular edge detection method $[31,33]$. For iris (or pupil) detection, the integrodifference values between the inner and outer boundaries of the iris (or pupil) were calculated in the input iris image with the changing radius values and the different positions of the iris (or pupil). The position and radius when the calculated integro-difference value was the maximum were determined as the detected iris or (pupil) position and radius. 
The upper and lower eyelids were also located by an eyelid detection mask and the parabolic eyelid detection method [33-35]. Since the eyelid line was regarded as a discontinuity area between the eyelid and iris regions, we first detected the eyelid candidate points by using an eyelid detection mask based on the first-order derivative. Because there were detection errors in the located candidate points, the parabolic Hough transform was applied to detect accurate positions of the eyelid line.

Then, we determined the eyelash candidate region based on the detected iris and pupil area and located the eyelash region $[33,36]$. The image focus was measured by the focus checking mask. Then, with the measured focus value of the input iris image, an eyelash-checking mask based on the first-order derivative was determined. If the image was defocused, a larger mask was used, and vice versa. The eyelash points were detected where the calculated value of the eyelash-checking mask was maximum and this was based on the continuous characteristics of the eyelash.

In circular edge detection, we did not use any threshold. By finding the position and radius with which the difference value was maximized, we were able to detect the boundaries of the pupil and the iris.

For eyelid detection masking and parabolic eyelid detection, we did not use any kind of threshold either. In the predetermined searching area as determined by the localized iris and pupil positions, the masking value of the eyelid detection mask was calculated vertically and the position with which the masking value was maximized was determined as the eyelid candidate position. Based on these candidate positions, we performed the parabolic Hough transform which had four control points: the curvature value of the parabola, the $X$ and $Y$ positions of the parabola apex, and the rotational angle of the parabola. In this case, because we detected one parabola with which the maximum value of curve fitting was obtained, we did not use any threshold. In order to reduce the processing time of the parabolic Hough transform, we restricted the searching dimensions of four control points by considering the conventional shape of the human eyelid.

For eyelash detection, because the eyelash points were detected on the maximum position, we again did not use any kind of user defined threshold.

After that, the detected circular iris region was normalized into rectangular polar coordinates $[1,37,38]$. In general, each iris image has variations in terms of the length of the outer and inner boundaries. The reason for these variations is that there are size variations between people's irises (the diameter of any iris can range from about 10.7-13 mm). Another reason is because the captured image size of any given iris may change according to the zooming factor caused by the $Z$-distance between the camera and the eye. Another reason is due to the dilation and contraction of the pupil (known as hippus movement).

In order to reduce these variations and obtain normalized iris images, we adjusted the lengths of the inner and outer iris boundaries to 256 pixels by stretching and linear interpolation. In conventional iris recognition, low, and mid-frequency components are mainly used for authentication instead of high-frequency information $[1,37,38]$. Con- sequently, linear interpolation did not degrade recognition accuracy. Experimental results with the captured iris images (400 images from 100 classes) showed that the accuracy of iris recognition when using linear interpolation was the same as when using bicubic interpolation and B-spline interpolation. So, we used linear interpolation to reduce processing time and system complexity.

Then, the normalized iris image was divided into 8 tracks and 256 sectors $[1,37,38]$. In each track and sector, the weighted mean of the gray level based on a 1D Gaussian kernel was calculated vertically $[1,37,38]$. By using the weighted mean of the gray level, we were able to reduce the effect caused by the iris segmentation error and obtain a 1D iris signal according to each track. We obtained eight $1 \mathrm{D}$ iris signals (256 pixels wide, resp., based on 256 sectors) from eight tracks. Consequently, we obtained a normalized iris region of $256 \times 8$ pixels, from 256 sectors and 8 tracks. Then, long and short Gabor filters were applied to generate the iris phase codes as shown in (6) [33],

$$
G(x)=A \cdot e^{-\pi\left[\left(x-x_{0}\right)^{2} / \sigma^{2}\right]}\left(\cos \left(2 \pi\left[u_{0}\left(x-x_{0}\right)\right]\right)\right),
$$

where $A$ is the amplitude of the Gabor filter, and $\sigma$ and $u_{0}$ are the kernel size and the frequency of the Gabor filter, respectively, [33].

Here, the long Gabor filter had a long kernel and was designed with a low frequency value. So, it passed a lowfrequency component of the iris textures. However, the short Gabor filter passed a mid-frequency component with a short kernel and a mid-frequency value for designing the Gabor kernel.

The optimal parameters of each Gabor filter were determined to obtain the minimum equal error rate (EER) by testing with test iris images. The EER is the error rate when the false acceptance rate (FAR) is the same as that of the false rejection rate (FRR). The FAR is the error rate of accepting imposter users as genuine ones. The FRR is the error rate of rejecting genuine users as imposters [33].

In terms of the long Gabor filter, the filter size was 25 pixels and the frequency $\left(u_{0}\right.$ of $\left.(6)\right)$ was $1 / 20$. In terms of the short Gabor filter, the filter size was 15 pixel and the frequency $\left(u_{0}\right.$ of $\left.(6)\right)$ was $1 / 16$. The calculated value of Gabor filtering was checked to determine whether it had a positive or negative value. If it had a positive value (including 0 ), the calculated value of Gabor filtering was 1 . If it had a negative value, it was $0[1,37,38]$. This was called iris code quantization and we used the iris phase information from that. The Gabor filter was applied on every track and sector, and we obtained an iris code of 2,048 bits ( $=256$ sectors $\times 8$ tracks) which had either a 1 or a 0 code. Consequently, 2,048 bits were obtained from long Gabor filtering and another 2,048 bits were obtained from short Gabor filtering [33].

In this case, the iris code bits which were extracted from the eyelid, eyelash, and SR occluded areas were regarded as unreliable and were not used for code matching [33]. After pupil, iris, eyelid, and eyelash detection, the noise regions were depicted as unreliable pixels (255). With Gabor filtering, even if one unreliable pixel was included in the range, the extracted bit on that position was regarded as an unreliable 
code bit. Only when the number of reliable codes exceeded the predetermined threshold (we used 1000 as the threshold to obtain the highest iris authentication accuracy with the iris database) they could be used as an enrolled template with high confidence [33].

The extracted iris code bits of the recognition image were compared with the enrolled template based on the hamming distance (HD) $[1,37,38]$. The HD was calculated based on the exclusive operation (XOR) between two code bits. So, if they were the same, the XOR value was 0 . If they were different, the value was 1 . Consequently, it was highly probable that the two iris codes of two genuine users would have both been 0 . Therefore, all the reliable code bits of the recognition image were compared with those of the enrolled one based on the HD. If the calculated HD exceeded the threshold (we used 0.3), the user was accepted as genuine. If not, he or she was rejected as an imposter.

\section{EXPERIMENTAL RESULTS}

Figure 5 shows the mobile phone that we used. It was a Samsung SPH-S2300 with a 2048*1536 pixel CCD sensor and a $3 \mathrm{X}$ optical zoom. To capture detailed iris patterns, we used IR-illuminators and an IR pass filter [19]. In front of the camera lens as shown in Figure 5, we attached a cold mirror (with an IR pass filter), which allowed IR light to pass through and reflect visible light. Also, we attached dual IRLED illuminators to detect genuine SRs easily (as mentioned in Section 2.2).

In the first test, we measured the accuracy (hit ratio) of our algorithm. Tests were performed on 400 face images captured from 100 persons (70 Asians, 30 Caucasians). These face images were not used for AdaBoost training. The test images consisted of the following four categories: images with glasses and contact lenses (100 images); images without glasses or contact lenses (100 images) in indoor environments (223 lux.); images with glasses and contact lenses (100 images); and images without glasses or contact lenses (100 images) in outdoor environments (1,394 lux.).

Experimental results showed that the pupil detection rate was $99.5 \%$ (for images without glasses or contact lenses in indoor and outdoor environments) and 99\% (for images with glasses or contact lenses in indoor and outdoor environments). The iris detection rate was $99.5 \%$ (for images without glasses or contact lenses in indoor and outdoor environments) and $98.9 \%$ (for images with glasses or contact lenses in indoor and outdoor environments). The detection rate was not degraded irrespective of conditions due to the illuminator mechanism as mentioned in Section 2.2. Though performance was slightly lower for users with glasses, contact lenses did not affect performance.

When we measured performance only using the AdaBoost algorithm, the detection rate was almost 98\%. But there were also many false alarms (e.g., when noneye regions such as eyebrows or frames of glasses were detected as correct eye regions). Experimental results with 400 face images showed that the false alarm rate using only the AdaBoost eye detector was almost $53 \%$. So, to solve these problems, we used both the information of the corneal SR and the Ad-

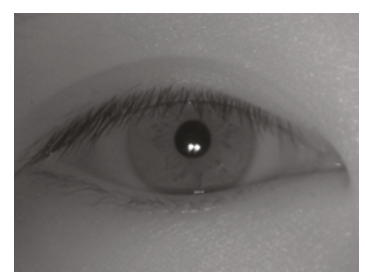

(a)

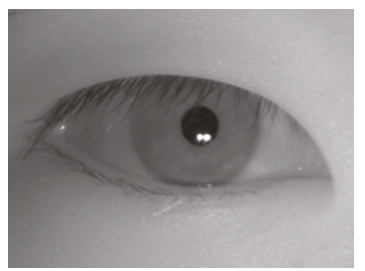

(b)
FIgURE 6: Examples of captured iris images.

aBoost eye detector. These results showed that the correct eye detection rate was more than $99 \%$ (as mentioned above) and the false alarm rate was less than $0.2 \%$.

Also, experimental results showed that the accuracies of the detected pupil (iris) center and radius were measured by the pixel RMS error between the detected and the manuallypicked ones. The RMS error of the detected pupil center was about 2.24 pixels ( 1 pixel on the $X$ axis and 2 pixels on the $Y$ axis, resp.). The RMS error of the pupil radius was about 1.9 pixels. Also, the results showed that the RMS error of the detected iris center was about 2.83 pixels (2 pixels on the $X$ axis and 2 pixels on the $Y$ axis, resp.). The RMS error of the iris radius was about 2.47 pixels. All the above localization accuracy figures were determined by manually assessing each image.

In the second test, we checked the correct detection rate of the pupil and the iris according to the size of the pupil detection box (as mentioned in Section 5) and as shown in Tables 1-4.

In the next experiments, we measured recognition accuracy with the captured iris images and detailed explanations of the recognition algorithm are presented in Section 6. Results showed that the EER was $0.05 \%$ when using 400 images (from 100 classes), which meant that the captured iris images could be used for iris recognition. Figure 6 and Table 5 show examples of the captured iris images and the FRR according to the FAR. In this case, the FAR refers to the error rate of accepting an imposter user as a genuine one, and the FRR refers to the error rate of rejecting a genuine user as an imposter. Here, an imposter means a user who did not enroll a biometric template in the database [33].

Then, we applied our iris recognition algorithm (as mentioned in Section 6) to the CASIA database version 1 [39] (using 756 iris images from 108 classes), the CASIA database version 3 [39] (a total of 22,051 iris images from more than 700 subjects), the iris images captured by our handmade iris camera based on the Quickcam Pro-4000 CCD camera [40] (using 900 iris images from 50 classes [33]) and those by the AlphaCam-I CMOS camera [41] (using 450 iris images from 25 classes [33]). Results showed that the iris authentication accuracies (EER) of the CASIA version 1, the CASIA version 3 , the iris images captured with the CCD camera, and the iris images captured with the CMOS camera were $0.072 \%$, $0.074 \%, 0.063 \%$, and $0.065 \%$, respectively. From that, it was clear that the authentication accuracy with the iris images captured by the mobile phone was superior and the captured iris images on mobile phone were of sufficient quality to be 
TABLE 1: Correct pupil detection rate for images without glasses (unit: \%).

\begin{tabular}{lcccc}
\hline & & \multicolumn{2}{c}{ The size of the pupil detection box } \\
& $120 * 120$ pixels & $140 * 140$ pixels & $160 * 160$ pixels & $180 * 180$ pixels \\
\hline $\begin{array}{l}\text { Correct detection rate } \\
\text { (hit ratio) }\end{array}$ & 90 & 95 & 99.5 & 100 \\
\hline
\end{tabular}

TABLE 2: Correct pupil detection rate for images with glasses or contact lenses (unit: \%).

\begin{tabular}{lcccc}
\hline & & \multicolumn{2}{c}{ The size of the pupil detection box } \\
& $120 * 120$ pixels & $140 * 140$ pixels & $160 * 160$ pixels & $180 * 180$ pixels \\
\hline $\begin{array}{l}\text { Correct detection rate } \\
\text { (hit ratio) }\end{array}$ & 87 & 94 & 99 & 100 \\
\hline
\end{tabular}

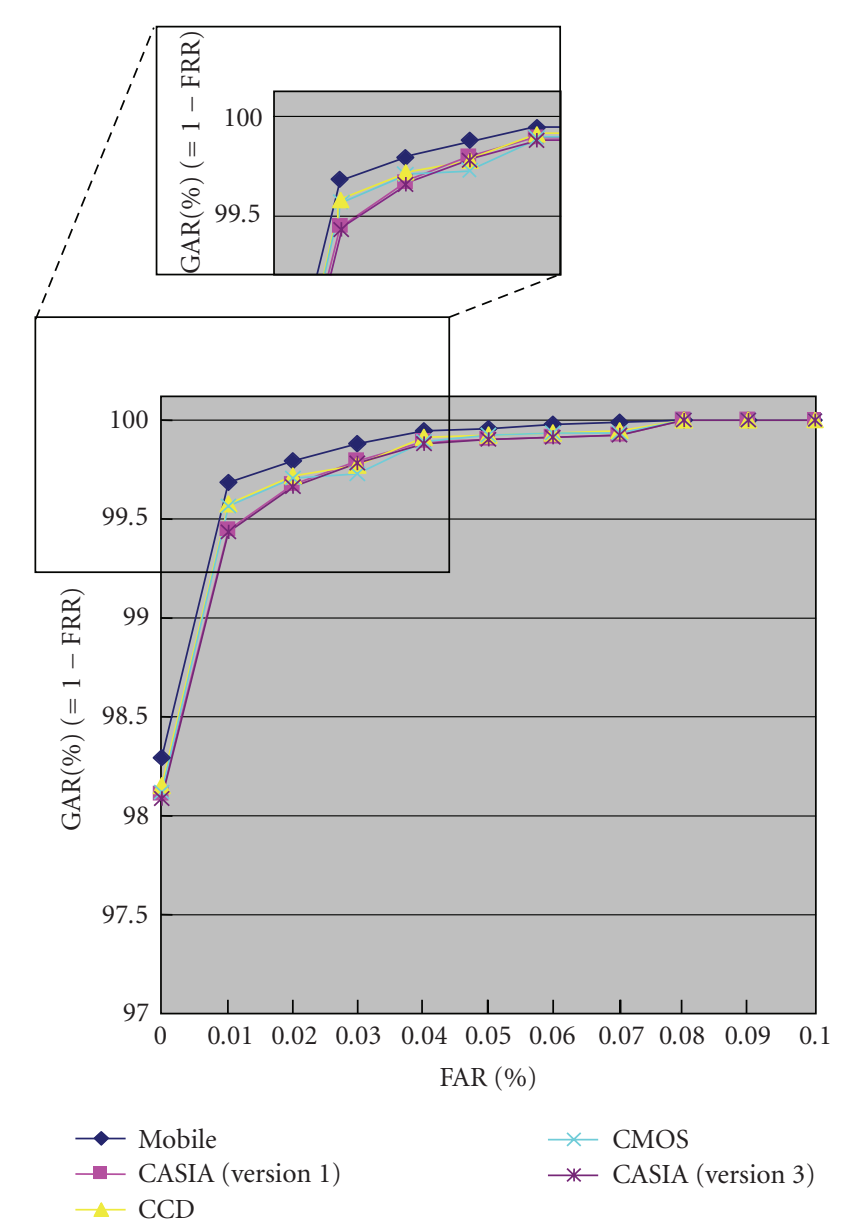

Figure 7: ROC curves for all datasets.

used for iris authentication. Figure 7 shows the ROC curves for the datasets such as the iris images obtained by our mobile phone camera, the CASIA version 1, the CASIA version 3, those by the Quickcam Pro-4000 CCD camera, and those by the AlphaCam-I CMOS camera.

In order to evaluate the robustness of our method to noise and show the degradation in the recognition accuracy as the amount of noise in the captured iris images increased, we increased the Gaussian noise in the iris images captured by our mobile phone camera. To measure the amount of inserted noise, we used the signal-to-noise rate $(\mathrm{SNR}=10 \times \log 10(\mathrm{Ps} / \mathrm{Pn}))$, where Ps represents the variance of the original image and Pn represents that of the noise image.

Results showed that if the SNR exceeded $10 \mathrm{~dB}$, there was no iris segmentation error or recognition. If the SNR was between 5-10 dB, the RMS error of the detected pupil and iris increased to $4.8 \%$ based on the original RMS error. However, even in that case, the recognition error was not increased. If the SNR was between 0 and $5 \mathrm{~dB}$, the RMS error of the detected pupil and iris increased to $6.2 \%$ based on the original RMS error. However, again, the recognition error was not increased.

That is because in conventional iris recognition, the lowand mid-frequency components of iris texture are mainly used for authentication instead of high-frequency information, as mentioned before $[1,33,37,38]$. Based on that, both long and short Gabor filters were applied to generate iris phase codes [33]. The long Gabor filter had a long kernel and was designed with a low frequency value (it passed the low-frequency component of the iris textures). Whereas, the short Gabor filter passed the mid-frequency component with a short kernel size and a mid-frequency value for designing the Gabor kernel.

In the next test, we measured different processing times with a mobile phone, a desktop PC, and a PDA. The mobile phone (SPH-S2300) used a Qualcomm MSM6100 chip (ARM926EJ-STM CPU (150 Mhz), 4 MB Memory) [28, 42]. To port our algorithm on the mobile phone, we used a wireless internet platform for interoperability (WIPI) 1.1 platform [43] without an additional DSP chip. For the PDA, we used an HP iPAQ hx4700 (with an Intel PXA270 CPU (624 Mhz), 135 MB Memory, and a Pocket PC 2003 (WinCE 4.2) OS). The desktop PC was a Pentium-IV CPU (3.2 Ghz), with 1 GB Memory and a Windows-XP OS.

Experimental results showed that the total processing times for iris detection and recognition in the desktop PC, PDA, and mobile phone were 29.32, 107.7, and 524.93 milliseconds, respectively. In previous research, the face detection algorithm proposed by Viola and Jones [44] was also tested on mobile phones such as the Nokia 7650 (with a CPU clock of $104 \mathrm{MHz}$ ) and the Sony-Ericsson P900 (with a CPU clock of $156 \mathrm{MHz}$ ) with an input image of $344 * 288$ pixels. Results showed that processing time on each 
TABLE 3: Correct iris detection rate for images without glasses (unit: \%).

\begin{tabular}{lcccc}
\hline & & \multicolumn{2}{c}{ The size of the pupil detection box } \\
& $120 * 120$ pixels & $140 * 140$ pixels & $160 * 160$ pixels & $180 * 180$ pixels \\
\hline $\begin{array}{l}\text { Correct detection rate } \\
\text { (hit ratio) }\end{array}$ & 90 & 94 & 99.5 & 99.8 \\
\hline
\end{tabular}

TABLE 4: Correct iris detection rate for images with glasses or contact lenses (unit: \%).

\begin{tabular}{lcccc}
\hline & & \multicolumn{3}{c}{ The size of the pupil detection box } \\
& $120 * 120$ pixels & $140 * 140$ pixels & $160 * 160$ pixels & $180 * 180$ pixels \\
\hline $\begin{array}{l}\text { Correct detection rate } \\
\text { (hit ratio) }\end{array}$ & 87 & 94.5 & 98.9 & 99.6 \\
\hline
\end{tabular}

TABLE 5: FRR according to FAR (unit: \%).

\begin{tabular}{ll}
\hline FAR & FRR \\
\hline 1.0 & 0.0 \\
0.1 & 0.0 \\
0.01 & 0.31 \\
0.001 & 1.59 \\
EER & 0.05 \\
\hline
\end{tabular}

mobile phone was 210 milliseconds and 160 milliseconds, respectively. Though these methods showed faster processing speed, they only included a face detection procedure and did not address recognition. In addition, they used an additional DSP chip, which increased their total costs.

\section{CONCLUSIONS}

In this paper, we have proposed a real-time pupil and iris detection method appropriate for mobile phones. This research has presented the following three advantages over previous works. First, for users with glasses, there may be many noncorneal SRs on the surface of the glasses and it is very difficult to detect genuine SRs on the cornea. To overcome these problems, we proposed the successive On/Off Scheme of the dual illuminators. Second, to detect SRs robustly, we proposed a theoretical way of estimating the size, shape, and brightness of SRs based on eye, camera, and illuminator models. Third, the detected eye (iris) regions by using the SRs were verified again by using the AdaBoost eye detector.

Results with 400 face images captured from 100 persons showed that the rate of correct iris detection was $99.5 \%$ (for images without glasses) and 98.9\% (for images with glasses and contact lenses). Consequent accuracy of iris authentication with 400 images from 100 classes was $0.05 \%$ of the equal error rate (EER) based on the detected iris image.

In future work, more field tests will be required. Also, to reduce processing time in mobile phones, we plan to port our algorithm into the ARM CPU of mobile phones. In addition, we plan to restore optical and motion blurred iris images and use them for recognition by not rejecting and recapturing images. This may reduce total processing time and enhance recognition accuracy.

\section{ACKNOWLEDGMENTS}

This work was supported by the Korea Science and Engineering Foundation (KOSEF) through the Biometrics Engineering Research Center (BERC) at Yonsei University.

\section{REFERENCES}

[1] J. G. Daugman, "High confidence visual recognition of persons by a test of statistical independence," IEEE Transactions on Pattern Analysis and Machine Intelligence, vol. 15, no. 11, pp. 1148-1161, 1993.

[2] American National Standards Institute Inc., "Initial Draft for Iris Image Format Revision ("Iris Image Interchange Format")," February 2007.

[3] A. L. Yuille, D. S. Cohen, and P. W. Hallinan, "Feature extraction from faces using deformable templates," in Proceedings of IEEE Computer Society Conference on Computer Vision and Pattern Recognition (CVPR '89), pp. 104-109, Rosemont, Ill, USA, June 1989.

[4] K.-M. Lam and H. Yan, "Locating and extracting the eye in human face images," Pattern Recognition, vol. 29, no. 5, pp. 771779, 1996.

[5] F. Zuo and P. H. N. de With, "Real-time face detection and feature localization for consumer applications," in Proceedings of the 4th PROGRESS Embedded Systems Symposium, pp. 257262, Utrecht, The Netherlands, October 2003.

[6] J. Rurainsky and P. Eisert, "Template-based eye and mouth detection for 3D video conferencing," in Visual Content Processing and Representation, vol. 2849 of Lecture Notes in Computer Science, pp. 23-31, Springer, Berlin, Germany, 2003.

[7] G. C. Feng and P. C. Yuen, "Multi-cues eye detection on gray intensity image," Pattern Recognition, vol. 34, no. 5, pp. 10331046, 2001.

[8] H. A. Rowley, S. Baluja, and T. Kanade, "Neural networkbased face detection," IEEE Transactions on Pattern Analysis and Machine Intelligence, vol. 20, no. 1, pp. 23-38, 1998.

[9] P. Viola and M. J. Jones, "Robust real-time face detection," International Journal of Computer Vision, vol. 57, no. 2, pp. 137154, 2004.

[10] Z. Zhu and Q. Ji, "Robust real-time eye detection and tracking under variable lighting conditions and various face orientations," Computer Vision and Image Understanding, vol. 98, no. 1, pp. 124-154, 2005.

[11] Y. Ebisawa and S.-I. Satoh, "Effectiveness of pupil area detection technique using two light sources and image difference method," in Proceedings of the 15th Annual International 
Conference of the IEEE Engineering in Medicine and Biology Society, pp. 1268-1269, San Diego, Calif, USA, October 1993.

[12] M. Suzaki, "Racehorse identification system using iris recognition," IEICE Transactions on Information and Systems, vol. J84D2, no. 6, pp. 1061-1072, 2001.

[13] Z. Ou, X. Tang, T. Su, and P. Zhao, "Cascade AdaBoost classifiers with stage optimization for face detection," in Proceedings of International Conference on Biometrics (ICB '06), vol. 3832 of Lecture Notes in Computer Science, pp. 121-128, Hong Kong, January 2006.

[14] Y. Freund and R. Schapire, "A short introduction to boosting," Journal of Japanese Society for Artificial Intelligence, vol. 14, no. 5, pp. 771-780, 1999.

[15] H. A. Park and K. R. Park, "A study on fast iris detection for iris recognition in mobile phone," Journal of the Institute of Electronics Engineers of Korea, vol. 43, no. 2, pp. 19-29, 2006.

[16] S. Han, H. A. Park, D. H. Cho, K. R. Park, and S. Y. Lee, "Face recognition based on near-infrared light using mobile phone," in Proceedings of the 8th International Conference on Adaptive and Natural Computing Algorithms (ICANNGA '07), Lecture Notes in Computer Science, pp. 11-14, Warsaw, Poland, April 2007.

[17] S. Rakshit and D. M. Monro, "Iris image selection and localization based on analysis of specular reflection," in Proceedings of IEEE Workshop on Signal Processing Applications for Public Security and Forensics (SAFE '07), Washington, DC, USA, April 2007.

[18] K. Choi, J.-S. Lee, and S.-J. Ko, "New autofocusing technique using the frequency selective weighted median filter for video cameras," IEEE Transactions on Consumer Electronics, vol. 45, no. 3, pp. 820-827, 1999.

[19] B. J. Kang and K. R. Park, "A study on iris image restoration," in Proceedings of the 5th International Conference on Audioand Video-Based Biometric Person Authentication (AVBPA '05), vol. 3546 of Lecture Notes in Computer Science, pp. 31-40, Hilton Rye Town, NY, USA, July 2005.

[20] American Conference of Government Industrial Hygienists, "Eye Safety with Near Infra-Red Illuminators," 1981.

[21] A. Gullstrand, "The optical system of the eye," in Physiological Optics, H. von Helmholtz, Ed., 3rd edition, 1909.

[22] R. C. Gonzalez, Digital Image Processing, Prentice-Hall, Englewood Cliffs, NJ, USA, 1992.

[23] http://www.polhemus.com/?page=Motion_Fastrak.

[24] P. Sandoz, D. Marsaut, V. Armbruster, P. Humbert, and T. Ghabi, "Towards objective evaluation of the skin aspect: principles and instrumentation," Skin Research and Technology, vol. 10, no. 4, pp. 263-270, 2004.

[25] E. C. Lee, K. R. Park, and J. Kim, "Fake iris detection by using purkinje image," in Proceedings of International Conference on Biometrics (ICB '06), vol. 3832 of Lecture Notes in Computer Science, pp. 397-403, Hong Kong, January 2006.

[26] R. L. Cook and K. E. Torrance, "Reflectance model for computer graphics," in Proceedings of the Annual Conference on Computer Graphics and Interactive Techniques (SIGGRAPH '81), pp. 307-316, Dallas, Tex, USA, August 1981.

[27] K. Shafique and M. Shah, "Estimation of the radiometric response functions of a color camera from differently illuminated images," in Proceedings of International Conference on Image Processing (ICIP '04), vol. 4, pp. 2339-2342, Singapore, October 2004.

[28] http://downloadcenter.samsung.com/content/UM/200411/ 20041126110547406_SPH-S2300_Rev3.0.pdf.

[29] Y. Freund and R. E. Schapire, "A decision-theoretic generalization of on-line learning and an application to boosting," Jour- nal of Computer and System Sciences, vol. 55, no. 1, pp. 119139, 1997.

[30] S.-W. Shih and J. Liu, "A novel approach to 3-D gaze tracking using stereo cameras," IEEE Transactions on Systems, Man, and Cybernetics, Part B: Cybernetics, vol. 34, no. 1, pp. 234-245, 2004.

[31] D.-H. Cho, K. R. Park, D. W. Rhee, Y. Kim, and J. Yang, "Pupil and iris localization for iris recognition in mobile phones," in Proceedings of the 7th ACIS International Conference on Software Engineering, Artificial Intelligence, Networking, and Parallel/Distributed Computing, Including the 2nd ACIS International Workshop on Self-Assembling Wireless Networks (SNPD/SAWN '06), vol. 2006, pp. 197-201, Las Vegas, Nev, USA, June 2006.

[32] D. H. Cho, K. R. Park, and D. W. Rhee, "Real-time iris localization for iris recognition in cellular phone," in Proceedings of the 6th International Conference on Software Engineering, Artificial Intelligence, Networking and Parallel/Distributed Computing and the 1st ACIS International Workshop on Self-Assembling Wireless Networks (SNPD/SAWN '05), vol. 2005, pp. 254-259, Towson, Md, USA, May 2005.

[33] H.-A. Park and K. R. Park, "Iris recognition based on score level fusion by using SVM," Pattern Recognition Letters, vol. 28, no. 15, pp. 2019-2028, 2007.

[34] Y. K. Jang, et al., "Robust eyelid detection for iris recognition," Journal of the Institute of Electronics Engineers of Korea, vol. 44, no. 1, pp. 94-104, 2007.

[35] Y. K. Jang, et al., "A study on eyelid localization considering image focus for iris recognition," submitted to Pattern Recognition Letters.

[36] B. J. Kang and K. R. Park, "A robust eyelash detection based on iris focus assessment," Pattern Recognition Letters, vol. 28, no. 13, pp. 1630-1639, 2007.

[37] J. Daugman, "How iris recognition works," IEEE Transactions on Circuits and Systems for Video Technology, vol. 14, no. 1, pp. 21-30, 2004.

[38] J. G. Daugman, "Demodulation by complex-valued wavelets for stochastic pattern recognition," International Journal of Wavelets, Multi-Resolution and Information Processing, vol. 1, no. 1, pp. 1-17, 2003.

[39] http://www.cbsr.ia.ac.cn/IrisDatabase.htm.

[40] http://www.logitech.com/index.cfm/webcam_communications/webcams/\&cl=us,en.

[41] http://www.avtech.co.kr/html/camera_etc.html.

[42] http://www.arm.com/products/CPUs/ARM926EJ-S.html.

[43] http://www.wipi.or.kr.

[44] http://www.idiap.ch/pages/contenuTxt/Demos/demo29/ face_finderfake.html. 